Article

\title{
Immunogenicity of a Heterologous Prime-Boost COVID-19 Vaccination with mRNA and Inactivated Virus Vaccines Compared with Homologous Vaccination Strategy against SARS-CoV-2 Variants
}

\author{
Ruiqi Zhang ${ }^{1}{ }^{\oplus}$, Danlei Liu ${ }^{1}$, Ka-Yi Leung ${ }^{2}{ }^{\oplus}$, Yujing Fan ${ }^{1}$, Lu Lu ${ }^{2}$, Pui-Chun Chan ${ }^{2}{ }^{\oplus}$, Kelvin Kai-Wang To ${ }^{2,3,4}$,

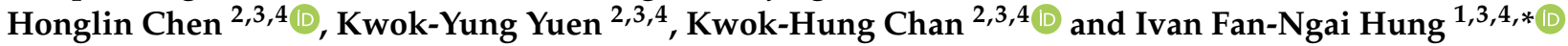 \\ check for \\ updates \\ 1 Department of Medicine, Li Ka Shing Faculty of Medicine, University of Hong Kong, Hong Kong Special \\ Administrative Region, Hong Kong 999077, China; zhangrq@hku.hk (R.Z.); danlei6@connect.hku.hk (D.L.); \\ jyjfan@connect.hku.hk (Y.F.) \\ 2 Department of Microbiology, Li Ka Shing Faculty of Medicine, University of Hong Kong, Hong Kong Special \\ Administrative Region, Hong Kong 999077, China; joy2u@connect.hku.hk (K.-Y.L.); \\ u3003963@connect.hku.hk (L.L.); bcpc@hku.hk (P.-C.C.); kelvinto@hku.hk (K.K.-W.T.); hlchen@hku.hk (H.C.); \\ kyyuen@hku.hk (K.-Y.Y.); chankh2@hku.hk (K.-H.C.) \\ 3 State Key Laboratory for Emerging Infectious Diseases, Li Ka Shing Faculty of Medicine, \\ University of Hong Kong, Hong Kong Special Administrative Region, Hong Kong 999077, China \\ 4 Carol Yu Centre for Infection, Li Ka Shing Faculty of Medicine, University of Hong Kong, Hong Kong Special \\ Administrative Region, Hong Kong 999077, China \\ * Correspondence: ivanhung@hku.hk
}

Citation: Zhang, R.; Liu, D.; Leung, K.-Y.; Fan, Y.; Lu, L.; Chan, P.-C.; To, K.K.-W.; Chen, H.; Yuen, K.-Y.; Chan, K.-H.; et al. Immunogenicity of a Heterologous Prime-Boost COVID-19 Vaccination with mRNA and Inactivated Virus Vaccines Compared with Homologous Vaccination Strategy against SARS-CoV-2 Variants. Vaccines 2022, 10, 72. https:// doi.org/10.3390/vaccines10010072

Academic Editor: Jorge H. Leitão

Received: 23 November 2021 Accepted: 30 December 2021

Published: 3 January 2022

Publisher's Note: MDPI stays neutral with regard to jurisdictional claims in published maps and institutional affiliations.

Copyright: (C) 2022 by the authors. Licensee MDPI, Basel, Switzerland. This article is an open access article distributed under the terms and conditions of the Creative Commons Attribution (CC BY) license (https:// creativecommons.org/licenses/by/ $4.0 /)$.

\begin{abstract}
The emergence of SARS-CoV-2 variants may impact the effectiveness of vaccines, while heterologous vaccine strategy is considered to provide better protection. The immunogenicity of an mRNA-inactivated virus vaccine against the SARS-CoV-2 wild-type (WT) and variants was evaluated in the study. SARS-CoV-2 naïve adults $(n=123)$ were recruited and placed in the following groups: BNT162b2, CoronaVac or BNT162b2-CoronaVac (Combo) Group. Blood samples were collected to measure neutralization antibodies $(\mathrm{NAb})$ by a live virus microneutralization assay (vMN) and surrogate NAb test. The day 56 vMN geometric mean titre (GMT) was 26.2 [95\% confident interval (CI), [22.3-30.9] for Combo, 136.9 (95\% CI, 104.2-179.7) for BNT162b2, and 14.7 (95\% CI, 11.6-18.6) for CoronaVac groups. At 6 months post-first dose, the GMT declined to 8.0, 28.8 and 7.1 in the Combo, BNT162b2 and CoronaVac groups, respectively. Three groups showed reduced neutralizing activity against D614G, beta, theta and delta variants. At day 56 GMT (74.6) and month 6 GMT (22.7), the delta variant in the BNT162b2 group was higher than that in the Combo (day 56, 7.4; month 6, 5.5) and CoronaVac groups (day 56, 8.0; month 6, 5) $(p<0.0001)$. Furthermore, the mean surrogate NAb value on day 56 in the BNT162b2 group was $594.7 \mathrm{AU} / \mathrm{mL}$ and higher than $40.5 \mathrm{AU} / \mathrm{mL}$ in Combo and 38.8 AU/mL in CoronaVac groups $(p<0.0001)$. None of the participants developed severe adverse events, and all other adverse events were self-limiting. The Combo vaccination strategy was safe. The overall vaccine immunogenicity at day 56 and 6 months were comparable to the homologous CoronaVac group but inferior to the homologous BNT162b2 group, against both the WT and all variants. Furthermore, the antibody response of vaccines waned at 6 months and thereby, a third dose of the vaccine is needed for these vaccines.
\end{abstract}

Keywords: COVID-19; heterologous vaccination; variants; neutralizing antibody

\section{Introduction}

The ongoing coronavirus disease 2019 (COVID-19) pandemic has lasted more than one and half years and continues to threaten the world. There are more than 250 million confirmed COVID-19 cases, including 5.2 million deaths [1]. COVID-19 is caused by 
the severe acute respiratory syndrome coronavirus 2 (SARS-CoV-2), and the vaccine is considered an effective way to contain the COVID-19 pandemic.

To date, there are seven vaccines recommended by World Health Organization (WHO) for emergency use, including four types of COVID-19 vaccines: mRNA, inactivated virus, viral vector-based and recombinant protein vaccine. BNT162b2, as the first mRNA vaccine approved by WHO, has shown high effectiveness and safety in clinical trials [1,2]. As a representative of inactivated virus vaccines, CoronaVac (Sinovac Life Sciences, Beijing, China) showed qualified immunogenicity in the phase 3 trial [3]. ChAdOx1(AZD1222) is a type of viral vector-based vaccine which looks to insert the SARS-CoV-2 spike protein gene into the chimpanzee adenovirus vector ChAdOx1 for replication, providing $70.4 \%$ vaccine effectiveness [4]. Although the recombinant protein vaccine NVX-CoV2373 (Novavax) has not been included in the emergency use list, it has been shown to be $89.7 \%$ effective in the clinical trial, and the efficacy against variant B.1.1.7 was approximately 86.3\% [5].

However, with the spread of the pandemic, the accumulation of mutations induces the emergence of several variants, which impact the effectiveness of COVID-19 vaccines [6-8]. A variant that detected a single mutant with D614G in the spike protein has shown a higher ability of transmission and replication $[9,10]$. Subsequently, several major variants appeared in some severely affected areas and spread rapidly around the world, including the B.1.1.7 (Alpha) variant found in the United Kingdom (UK), B.1.351 (Beta) variant found in South Africa, P.1 (Gamma) variant found in Brazil and B.1.617.2 (Delta) in India. These variants accumulated multiple mutations and involved the spike protein [10,11]. Since most vaccine development uses the spike protein as an antigen, the mutations of the SARS-CoV-2 in the spike protein make the efficacy of the vaccine a concern. Some studies have examined the protection of vaccinations against variants in a population. The results showed that the vaccine is still effective against the current variants, but its effectiveness is weakened in the infected patients $[10,12-14]$. The mutation of the virus makes the prevention situation serious, and changes in vaccine strategies will continue to be monitored.

Meanwhile, the current situation of the shortage of vaccines in the world cannot be ignored. As an alternative, the possibility of sequentially administering different SARS-CoV-2 vaccines, known as heterologous schedules, could be an opportunity to make vaccination programs more flexible and reliable in the face of supply fluctuations. The strategy of heterologous prime-boost is considered to combine the best characteristic of each vaccine to enhance the immune response and may show better protection when the virus mutates; this kind of strategy has been used to protect against Ebola $[15,16]$. Just as some countries have recommended the use of mRNA vaccines for heterologous booster immunization after initial immunization with the ChAdOx1 vaccine, it has been proven to be a safe and effective strategy in the clinical trial [17].

The aim of this study was to explore the immunogenicity of a heterologous primeboost COVID-19 vaccination with mRNA and inactivated virus vaccines and compare it with homologous vaccination. The result may provide new thinking for the current vaccine situation.

\section{Materials and Methods}

\subsection{Study Design and Participants}

This study was an open trial completed in two vaccination centres in Hong Kong. Healthy adults aged 18-60 years with no related infectious history were invited and recruited into the study. We informed the participants of the related precautions before the study and obtained their informed consent. The study was approved by the institutional review board of the University of Hong Kong and Hospital Authority (UW 21-214).

\subsection{Procedures}

The study was divided into three groups; participants in each group were vaccinated with different types of SARS-CoV-2 vaccine, including the BNT162b2, CoronaVac, and Combo (BNT162b2- CoronaVac) vaccine. In the BNT162b2 group, first and second doses 
of COVID-19 vaccines were vaccinated at day 0 and day 21 with the homologous vaccine, while the CoronaVac groups were vaccinated on day 0 and day 28 . In the Combo vaccine group, participants received heterologous BNT162b2 and CoronaVac vaccines on day 0 and day 28, respectively. The study did not set a random mechanism and did not blind the participants and investigators. The participants could choose the type of vaccination according to their personal wishes and were notified of the collection of blood at baseline, day 21 (BNT162b2) or day 28 (CoronaVac and Combo vaccine), day 56 and month 6 (Figure 1).

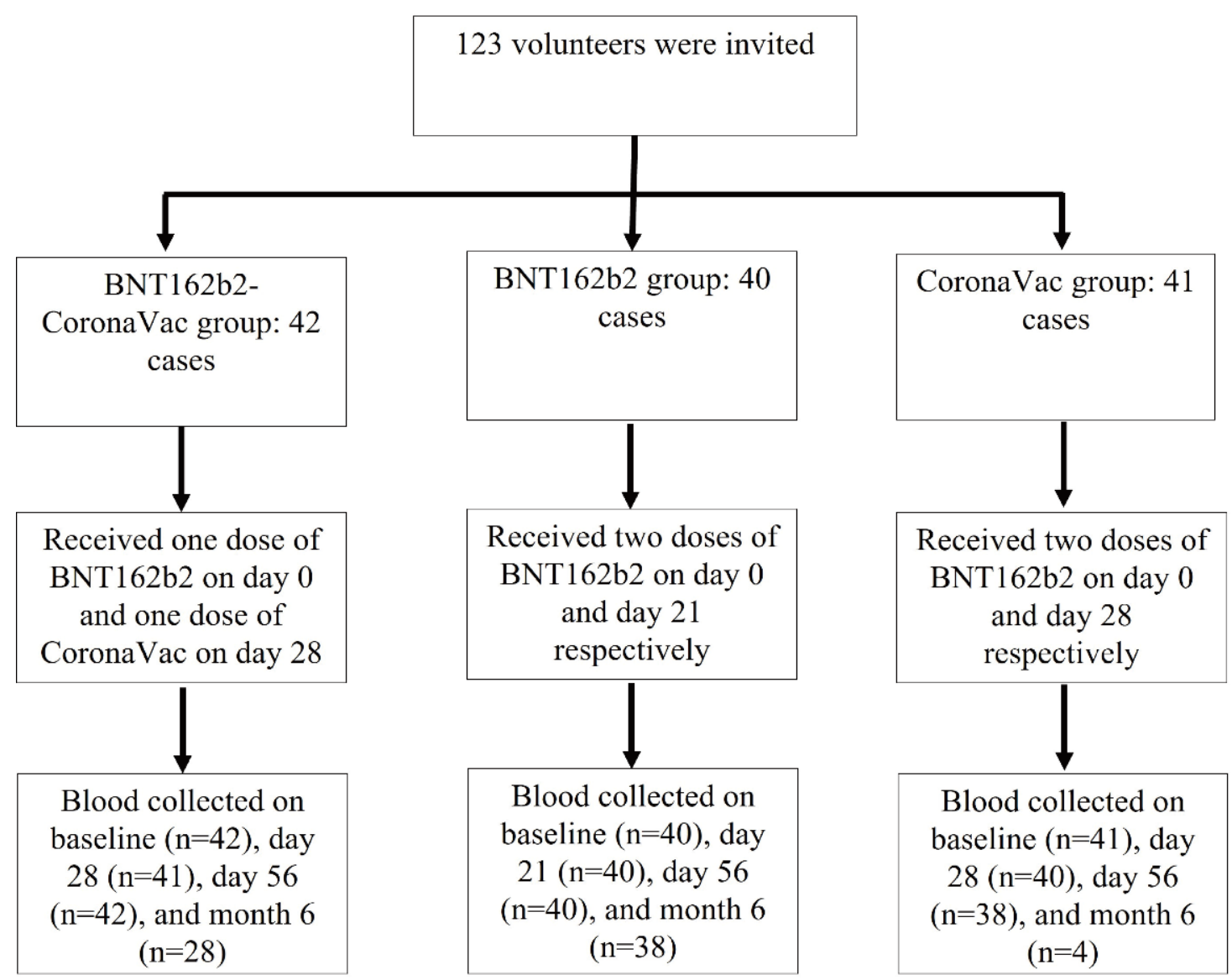

Figure 1. Participant recruitment and research flow diagram.

A live virus microneutralization (vMN) assay was performed in the Biosafety Level 3 facility at HKU to compare the effectiveness of antibody levels against different SARSCoV-2 strains, including the HKU-001a (wild type, GenBank accession number MT230904) strain (WT), B.1.36.27 (D614G), B.1.1.7 (alpha variant), B.1.351 (beta variant), P.3. (theta variant) and B.1.617.2 (delta variant) [18]. The serum samples were treated at $56{ }^{\circ} \mathrm{C}$ for 30 min before testing. VeroE6 TMPRSS2 cells (JCRB Cell Bank Catalogue no. JCRB1819) were seeded in a 96-well plate and incubated at $37{ }^{\circ} \mathrm{C}$ and $5 \% \mathrm{CO}_{2}$. A serial two-fold dilution was performed and started at 1:10 for the serum. One hundred $50 \%$ tissue culture infective dose (TCID50) of SARS-CoV-2 virus was mixed with serum and incubated for $1.5 \mathrm{~h}$ at $37^{\circ} \mathrm{C}$, the mixture was added to the cells and placed in the $37^{\circ} \mathrm{C}$ for $72 \mathrm{~h}$ incubation. Cytopathic effect (CPE) was observed and recorded at $72 \mathrm{~h}$. The titre of vMN was defined as the maximum dilution of serum in which the percentage of CPE is equal to or less than $50 \%$. vMN titre $\geq 10$ was considered as positive.

Surrogate neutralizing antibody (NAb) against the SARS-CoV-2 RBD and anti-nucleocapsid protein (N) IgG was determined with chemiluminescent microparticle immunoassay on the iFlash 1800 analyzer (Shenzhen YHLO Biotech Co., Ltd., Shenzhen, China) [18]. In the sample loading area, sera were placed on a sample rack, and then the iFlash system performed the test automatically. For surrogate NAb, the cut-off value for seropositivity 
was $15 \mathrm{AU} / \mathrm{mL}$, and the maximum measurable value was $800 \mathrm{AU} / \mathrm{mL}$. For the anti-N IgG, the cut-off value for seropositivity was $10 \mathrm{AU} / \mathrm{mL}$

\subsection{Outcomes}

The primary end-point was titre. The secondary end-point was geometric mean titre (GMT) fold increase and the rate of adverse events. For assessing safety, participants were informed of the vaccine name, risks and precautions and were asked to observe severe adverse events for half an hour after vaccination. All participants were given a symptom diary to record the adverse effects 4 weeks after each dose. The adverse events contain system reactions and local reactions. Systemic reactions included fever, headache, muscle pain, skin rash, joint pain, vomit, tiredness, diarrhea, chills and severe adverse events (SAE). SAE were defined as vaccine-related undesired events, including death, disability or life-threatening conditions. Pain at the injection site, itching, swelling, redness, and ecchymosis were listed as local reactions.

\subsection{Statistics}

Statistical inference of continuous variables was performed using one-way ANOVA and analysis of variance, least-significant difference (LSD) multiple comparison was used when meeting the homogeneity of variance, otherwise Dunnett's multiple comparison was used, including demographic parameters and antibody level. Pearson chi-square and Fisher's exact test were utilized for numerical or categorical data. SPSS 27.0 was used for statistical computation. Results all used a two-tailed test and were considered significant at $p<0.05$.

\section{Results}

\subsection{Subjects}

Between May and November 2021, 122 SARS-CoV-2 naïve individuals were invited to the participate in the COVID-19 vaccination programme. Forty-two individuals received one dose of BNT162b2 followed by one dose of CoronaVac (median age $=44.5$ years), 40 individuals received two doses of BNT162b2 (median age $=49$ years) and 41 individuals received two doses of CoronaVac (median age $=47$ years) $($ Table 1$)$.

Table 1. Demographic and clinical baseline characteristics.

\begin{tabular}{ccccc}
\hline & Combo $(\boldsymbol{n}=\mathbf{4 2})$ & CoronaVac $(\boldsymbol{n}=\mathbf{4 1})$ & BNT162b2 $(\boldsymbol{n}=\mathbf{4 0})$ & $\boldsymbol{p}^{\mathbf{2}}$ \\
\hline Median age (years) & $44.5(36-50.5)^{1}$ & $49(39.5-54.5)$ & $47(33-51.75)$ & 0.232 \\
Male/Female (\%) & $24 / 18(57.1 / 42.8)$ & $13 / 28(31.7 / 68.3)$ & $19 / 21(47.5 / 52.5)$ & 0.065 \\
\hline
\end{tabular}

${ }^{1}$ data were median age (IQR). ${ }^{2} p<0.05$, the results are significantly different.

\subsection{Immunogenicity of Vaccines Determined by $v M N$}

The results of NAb in sera determined by vMN showed that these participants had no antibodies against WT on the baseline. On day 28 , individuals from the Combo group had a higher geometric mean titre (GMT) [23.7, 95\% confidence interval (CI), 18.8-29.9] against WT than CoronaVac groups (5.6, 95\% CI, 5.1-6.2) $(p<0.0001)$ (Table 2) (Figure 2a). In the BNT162b2 group, day 21 vMN GMT was 13.4 (95\% CI, 9.7-18.7) (Table 2). The vMN GMT on day 56 increased to 26.2 (95\% CI, 22.3-30.9), 136.9 (95\% CI, 104.2-179.7) and 14.7 (95\% CI, 11.6-18.6) in the Combo, BNT162b2 and CoronaVac groups, respectively (Table 2). The GMT fold increase on day 56 was 5.2 (95\% CI, 4.5-6.2) in Combo, 27.4 (95\% CI, 20.8-36.0) in BNT162b2, and 2.9 (95\% CI, 2.3-3.7) in the CoronaVac group. On day 56, GMT in the BNT162b2 group was significantly higher than that in the Combo $(p<0.0001)$ and CoronaVac groups $(p<0.0001)$, while no statistically significant difference was observed in GMT between the Combo and CoronaVac groups (Figure 2a). At 6 months post-first dose of vaccine, the GMT declined compared with that on day 56 and were $8.0(95 \% \mathrm{CI}, 6.6-9.8)$, 28.8 (95\% CI, 22.3-37.2) and 7.1 (95\% CI, 4.8-10.5) in the Combo, BNT162b2 and CoronaVac groups, respectively (Table 2). 
Table 2. Immunogenicity of different vaccination strategies.

\begin{tabular}{|c|c|c|c|c|}
\hline & Combo $(n=42)$ & BNT162b2 $(n=40)$ & CoronaVac $(n=41)$ & $p^{9}$ \\
\hline \multicolumn{5}{|l|}{ WT } \\
\hline \multicolumn{5}{|l|}{ Baseline } \\
\hline $\mathrm{GMT}^{1}$ & $5(5-5)$ & $5(5-5)$ & $5(5-5)$ & \\
\hline \multicolumn{5}{|l|}{ Day $21^{2} /$ Day 28} \\
\hline GMT & $23.7(18.8-29.9)$ & $13.4(9.7-18.7)$ & $5.6(5.1-6.2)$ & 0.002 \\
\hline GMT fold increase & $4.7(3.8-6.0)^{3}$ & $2.7(1.9-3.7)$ & $1.1(1.0-1.2)^{6}$ & 0.002 \\
\hline \multicolumn{5}{|l|}{ Day 56} \\
\hline GMT & $26.2(22.3-30.9)$ & $136.9(104.2-179.7)$ & $14.7(11.6-18.6)$ & $<0.0001$ \\
\hline GMT fold increase & $5.2(4.5-6.2)$ & $27.4(20.8-36.0)$ & $2.9(2.3-3.7)^{7}$ & $<0.0001$ \\
\hline \multicolumn{5}{|l|}{ Month 6} \\
\hline GMT & $8.0(6.6-9.8)$ & $28.8(22.3-37.2)$ & 7.1(4.8-10.5) & $<0.0001$ \\
\hline GMT fold increase & $1.6(1.3-2.0)^{4}$ & $5.8(4.5-7.4)^{5}$ & $1.4(1.0-2.1)^{8}$ & $<0.0001$ \\
\hline \multicolumn{5}{|l|}{ D614G } \\
\hline \multicolumn{5}{|l|}{ Baseline } \\
\hline GMT & $5(5-5)$ & $5(5-5)$ & $5(5-5)$ & \\
\hline \multicolumn{5}{|l|}{ Day 21/Day 28} \\
\hline GMT & $9.0(7.5-11.0)$ & $9.0(7.3-11.2)$ & $5.2(4.8-5.5)$ & 0.050 \\
\hline GMT fold increase & $1.8(1.5-2.2)$ & $1.8(1.5-2.2)$ & $1.0(1.0-1.1)$ & 0.050 \\
\hline \multicolumn{5}{|l|}{ D614G } \\
\hline \multicolumn{5}{|l|}{ Day 56} \\
\hline GMT & $13.1(10.7-16.1)$ & $81.4(60.0-110.5)$ & $11.8(9.6-14.5)$ & $<0.0001$ \\
\hline GMT fold increase & $2.6(2.1-3.2)$ & $16.3(12.0-22.1)$ & $2.4(1.9-2.9)$ & $<0.0001$ \\
\hline \multicolumn{5}{|l|}{ Month 6} \\
\hline GMT & $5.7(5.0-6.4)$ & $21.5(17.0-27.2)$ & $5(5-5)$ & $<0.0001$ \\
\hline GMT fold increase & $1.1(1.0-1.3)$ & $4.3(3.4-5.4)$ & $1.0(1.0-1.0)$ & $<0.0001$ \\
\hline \multicolumn{5}{|l|}{ Alpha } \\
\hline \multicolumn{5}{|l|}{ Baseline } \\
\hline GMT & $5(5-5)$ & $5(5-5)$ & $5(5-5)$ & \\
\hline \multicolumn{5}{|l|}{ Day 21/Day 28} \\
\hline GMT & $7.5(6.1-9.2)$ & $9.0(7.1-11.5)$ & $5.4(4.9-5.9)$ & 0.177 \\
\hline GMT fold increase & $1.5(1.2-1.8)$ & $1.8(1.4-2.3)$ & $1.1(1.0-1.2)$ & 0.177 \\
\hline \multicolumn{5}{|l|}{ Day 56} \\
\hline GMT & $11.5(8.9-14.7)$ & 146.7 (106.2-202.7) & $11.6(9.0-15.0)$ & $<0.0001$ \\
\hline GMT fold increase & $2.3(1.8-2.9)$ & $29.3(21.2-40.5)$ & $2.3(1.8-3.0)$ & $<0.0001$ \\
\hline \multicolumn{5}{|l|}{ Month 6} \\
\hline GMT & $6.6(5.5-7.8)$ & $37.9(27.7-51.8)$ & $7.1(4.8-10.5)$ & $<0.0001$ \\
\hline GMT fold increase & $1.3(1.1-1.6)$ & $7.6(5.5-10.4)$ & $1.4(1.0-2.1)$ & $<0.0001$ \\
\hline \multicolumn{5}{|l|}{ Beta } \\
\hline \multicolumn{5}{|l|}{ Baseline } \\
\hline GMT & $5(5-5)$ & $5(5-5)$ & $5(5-5)$ & \\
\hline \multicolumn{5}{|l|}{ Day 21/Day 28} \\
\hline GMT & $5(5-5)$ & $5(5-5)$ & $5.2(4.8-5.5)$ & 0.366 \\
\hline GMT fold increase & $1.0(1.0-1.0)$ & $1.0(1.0-1.0)$ & $1.0(1.0-1.1)$ & 0.366 \\
\hline \multicolumn{5}{|l|}{ Day 56} \\
\hline GMT & $5.4(4.8-5.9)$ & $15.7(12.0-20.5)$ & $5(5-5)$ & $<0.0001$ \\
\hline GMT fold increase & $1.1(1.0-1.2)$ & $3.1(2.4-4.1)$ & $1.0(1.0-1.0)$ & $<0.0001$ \\
\hline \multicolumn{5}{|l|}{ Month 6} \\
\hline GMT & $5(5-5)$ & $12.2(9.8-15.2)$ & $5(5-5)$ & $<0.0001$ \\
\hline GMT fold increase & $1.0(1.0-1.0)$ & $2.4(2.0-3.0)$ & $1.0(1.0-1.0)$ & $<0.0001$ \\
\hline
\end{tabular}


Table 2. Cont.

\begin{tabular}{|c|c|c|c|c|}
\hline & Combo $(n=42)$ & BNT162b2 $(n=40)$ & CoronaVac $(n=41)$ & $p^{9}$ \\
\hline \multicolumn{5}{|l|}{ Theta } \\
\hline \multicolumn{5}{|l|}{ Baseline } \\
\hline GMT & $5(5-5)$ & $5(5-5)$ & $5(5-5)$ & \\
\hline \multicolumn{5}{|l|}{ Day 21/Day 28} \\
\hline GMT & $5.4(5.0-5.7)$ & $6.7(6.0-7.6)$ & $5.2(4.8-5.5)$ & 0.001 \\
\hline GMT fold increase & $1.1(1.0-1.1)$ & $1.3(1.2-1.5)$ & $1.0(1.0-1.1)$ & 0.001 \\
\hline \multicolumn{5}{|l|}{ Day 56} \\
\hline GMT & $5.9(5.2-6.8)$ & $39.3(27.6-55.9)$ & $7.1(5.9-8.6)$ & $<0.0001$ \\
\hline GMT fold increase & $1.2(1.0-1.4)$ & $7.9(5.5-11.2)$ & $1.4(1.2-1.7)$ & $<0.0001$ \\
\hline \multicolumn{5}{|l|}{ Theta } \\
\hline \multicolumn{5}{|l|}{ Month 6} \\
\hline GMT & $5.3(4.9-5.6)$ & $14.4(11.5-18.1)$ & $5(5-5)$ & $<0.0001$ \\
\hline GMT fold increase & $1.1(1.0-1.1)$ & $2.9(2.3-3.6)$ & $1.0(1.0-1.0)$ & $<0.0001$ \\
\hline \multicolumn{5}{|l|}{ Delta } \\
\hline \multicolumn{5}{|l|}{ Baseline } \\
\hline GMT & $5(5-5)$ & $5(5-5)$ & $5(5-5)$ & \\
\hline \multicolumn{5}{|l|}{ Day 21/Day 28} \\
\hline GMT & $5.9(5.3-6.6)$ & $8.6(7.1-10.3)$ & $5.7(5.0-6.5)$ & 0.008 \\
\hline GMT fold increase & $1.2(1.1-1.3)$ & $1.7(1.4-2.1)$ & $1.1(1.0-1.3)$ & 0.008 \\
\hline \multicolumn{5}{|l|}{ Day 56} \\
\hline GMT & $7.4(6.2-8.8)$ & $74.6(55.4-100.7)$ & $8.0(6.6-9.8)$ & $<0.0001$ \\
\hline GMT fold increase & $1.5(1.2-1.8)$ & $14.9(11.1-20.1)$ & $1.6(1.3-2.0)$ & $<0.0001$ \\
\hline \multicolumn{5}{|l|}{ Month 6} \\
\hline GMT & $5.5(5.0-6.1)$ & 22.7 (17.3-29.9) & $5(5-5)$ & $<0.0001$ \\
\hline GMT fold increase & $1.1(1.0-1.2)$ & $4.5(3.5-6.0)$ & $1.0(1.0-1.0)$ & $<0.0001$ \\
\hline
\end{tabular}

1: data are mean values $(95 \% \mathrm{CI}){ }^{2}$ : the blood samples of the BNT162 group collected on day $21 .{ }^{3}: 41$ individuals collected blood samples on day $28 .{ }^{4}: 28$ individuals collected blood samples at 6 months. ${ }^{5}: 38$ individuals collected blood samples at 6 months. ${ }^{6}: 40$ individuals collected blood samples on day $28 .{ }^{7}: 38$ individuals collected blood samples on day 56. ${ }^{8}: 4$ individuals collected blood samples at 6 months. ${ }^{9}: p<0.05$, the results are significantly different.

Besides WT, vMN titres against variants (D614G, alpha, beta, theta, delta) were also determined. For the D614G variant, the BNT162b2 group showed significantly higher results on day 56 GMT $(81.4,95 \%$ CI, 60.0-110.5) $(p<0.0001)$ than the Combo $(13.1,95 \% \mathrm{CI}$, 10.7-16.1) and CoronaVac groups (11.8, 95\% CI, 9.6-14.5) (Table 2) (Figure 2b). At 6 months, the GMT against D614G in the Combo, BNT162b2 and CoronaVac groups reduced to 5.7 (95\% CI, 5.0-6.4), 21.5 (95\% CI, 17.0-27.2) and 5 (95\% CI, 5-5), respectively. After full vaccination, there was no statistically significant difference in GMT at day 56 and month 6 between the Combo and CoronaVac groups (Figure 2b). For the alpha variant, individuals in the BNT162b2 group showed higher GMT on day $56(146.7)(p<0.0001)$ and month 6 (37.9) $(p<0.0001)$ than these from the Combo group (day 56 GMT, 11.5; month 6 GMT, 6.6) and the CoronaVac group (day 56 GMT, 11.6; month 6 GMT, 7.1), while the Combo group showed a comparable antibody response at day 56 and month 6 with CoronaVac group (Table 2) (Figure 2c). 


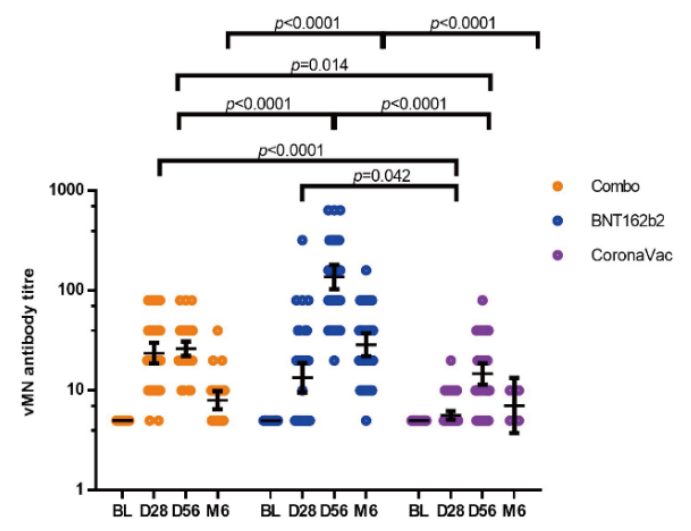

c

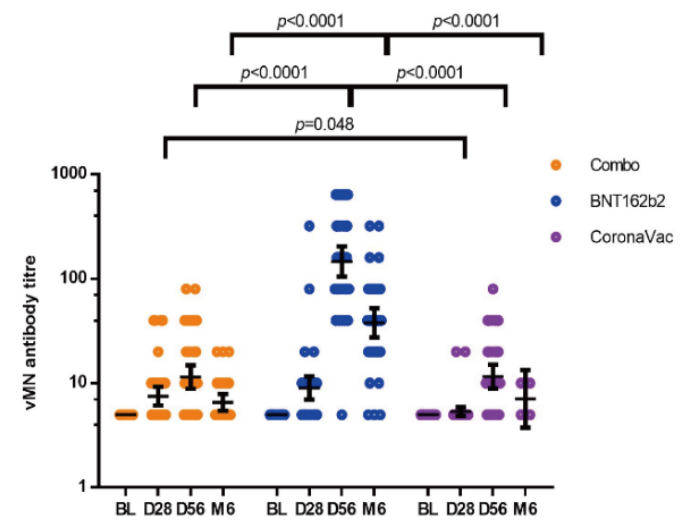

e

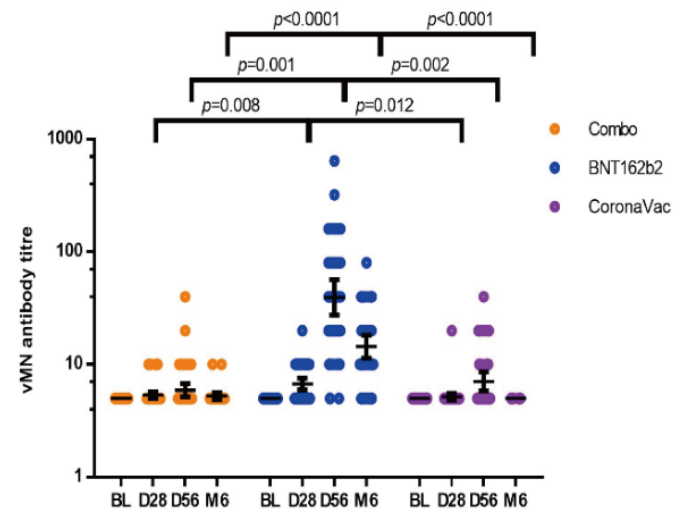

$\mathrm{b}$

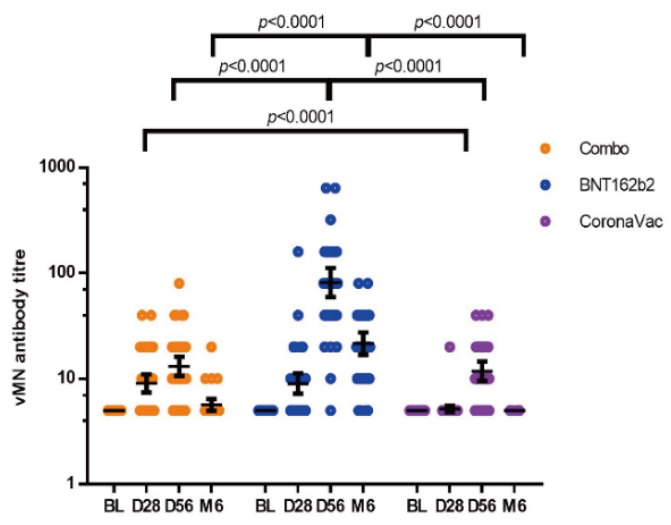

d

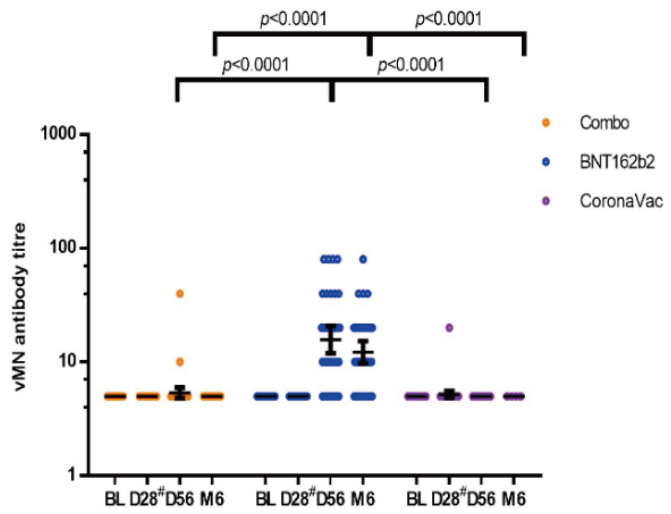

f

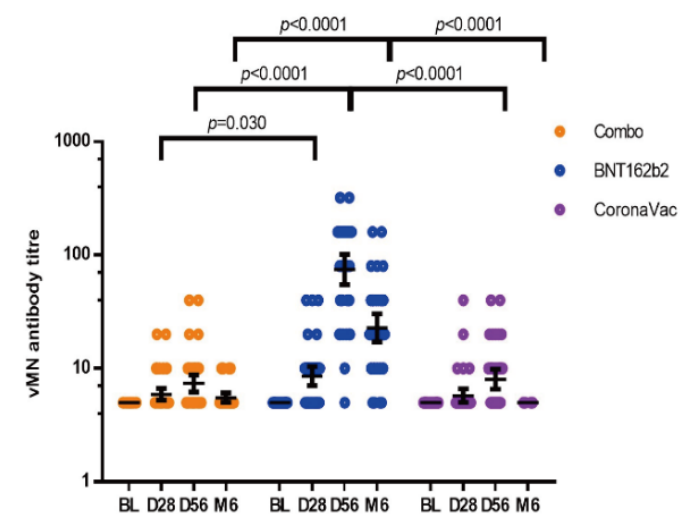

Figure 2. Comparison of immunogenicity of three vaccination strategies. SARSA-CoV-2 naïve individuals were invited to participate in the vaccination programme. In the Combo group, subjects received one dose of BNT162b2 on day 0 and one dose of CoronaVac on day 28. Then, blood samples were collected on the baseline, day 28, day 56 and month 6 . For BNT162b2, subjects recieved two doses of BNT162b2 at the baseline and day 21 respectively and blood was taken at the baseline, day 21, day 56 and month 6 . For CoronaVac, Individuals received two doses of CoronaVac vaccine at the 
baseline and day 28, respectively, and then blood was taken at the baseline, day 28, day 56 and month 6. The live virus microneutralization assay (vMN) was used to determine the level of neutralizing antibodies in serum against (a) wild type, (b) D614G, (c) alpha, (d) beta, (e) theta and (f) delta. BL: baseline; D28: day 28 for Combo and CoronaVac, day 21 for BNT162b2; D56: day 56; M6: month 6. To analyse the difference in immunogenicity between two groups, a post hoc multiple comparison was performed, and a $p$ value was shown on the graph if the value was less than 0.05 , which represents a statistically significant difference.

Vaccines showed low effectiveness against the beta variant. On day 56, the GMT was 5.4 (95\% CI, 4.8-5.9) against the beta variant in the Combo group, and 15.7 (95\% CI, 12.0-20.5) in BNT162b2 and 5 (95\% CI, 5-5) in the CoronaVac group (Table 2). The GMT on day 56 in the BNT162b2 group was higher than that in the Combo $(p<0.0001)$ and CoronaVac $(p<0.0001)$ groups and no statistically significant difference was observed in GMT between the Combo and CoronaVac groups (Figure $2 \mathrm{~d}$ ). These vaccination strategies also showed low effectiveness against the theta variant, and the day 56 GMT in the Combo group was 5.9 (95\% CI, 5.2-6.8), which was lower than that in BNT162b2 (day 56 GMT, 39.3; 95\% CI, 27.6-55.9) $(p<0.0001)$ and comparable with the CoronaVac group (day 56 GMT, 7.1; 95\%CI, 5.9-8.6) (Table 2) (Figure 2e). For the delta variant, the day 56 GMT and month 6 GMT in the BNT162b2 group (day 56, 74.6; month 6, 13) was higher than that in the Combo (day 56, 7.4; month 6, 5.5) and CoronaVac groups (day 56, 8.0; month 6, 5) $(p<0.0001)$ (Table 2) (Figure 2f). When comparing the immunogenicity against delta between the Combo and CoronaVac groups, no statistically significant difference was observed at day 56 and month 6 (Figure 2f). Overall, the BNT162b2 platform showed the highest immunogenicity, and the Combo platform induced comparable antibody response with CoronaVac groups against WT and five variants.

\subsection{Immunogenicity of Vaccines Determined by Surrogate NAb Test}

In addition to the vMN assay, chemiluminescent microparticle immunoassay, which is a type of surrogate NAb test, was used to determine the antibody response induced by vaccines. Similar to the results produced by vMN, the mean surrogate NAb value was 37.1 AU/mL for the Combo group, 70.6 AU/mL for the BNT162b2 group, and $5.5 \mathrm{AU} / \mathrm{mL}$ for the CoronaVac group post first dose of vaccine (Table 3). On day 56, individuals from the Combo group showed a slight increase in the surrogate Nab value (mean, $40.5 \mathrm{AU} / \mathrm{mL}$ ), while the mean of the surrogate NAb values in the other two groups jumped to $594.7 \mathrm{AU} / \mathrm{mL}(\mathrm{BNT162b})$ and $38.8 \mathrm{AU} / \mathrm{mL}$ (CoronaVac). Then, at 6 months, there was a decrease of the surrogate $\mathrm{NAb}$ level, and the mean was $8.6 \mathrm{AU} / \mathrm{mL}$ in Combo, 138.5 AU/mL in BNT162b2 and 9.2 AU/mL in the CoronaVac group (Table 3). Furthermore, anti-N IgG was also tested. Only the CoronaVac group showed anti-N IgG on day 56, and the mean value was 21.8 AU/mL (Table 3).

\subsection{Safety}

After a primer dose of vaccine, the incidence of total local symptoms in the BNT162b2 and Combo groups was significantly higher than that of CoronaVac (Combo, 97.6\%; BNT162b2, 81.8\%; CoronaVac, 34.3\%; $p<0.0001)$, and there was no significant difference in total systemic reactions between three groups (Combo, 45.2\%; BNT162b2, 45.5\%; CoronaVac, $37.1 \% ; p=0.761)$. The second dose of the vaccine induced higher frequencies of systemic reactions $(66.7 \%)(p<0.0001)$ and local reactions $(75.8 \%)(p<0.0001)$ in the BNT162b2 group than in the Combo (systemic reactions, 31.0\%; local reactions, 35.7\%) and CoronaVac groups (systemic reactions, 22.9\%; local reactions, 20.0\%) (Table 4). The most common symptom induced by the second dose of COVID-19 vaccine in three groups was injection site pain (Combo, 35.7\%; BNT162b2, 69.7\%; CoronaVac, 20.0\%). After the second dose, the frequencies of chill, tiredness, muscle pain, joint pain, redness, swelling and itching in the BNT162b2 group were higher than those in the Combo and CoronaVac groups (Table 4). No SAE was observed in the study after vaccination. 
Table 3. Surrogate NAb and anti-N IgG.

\begin{tabular}{|c|c|c|c|c|}
\hline & Combo $(n=42)$ & BNT162b2 $(n=40)$ & CoronaVac $(n=41)$ & $p^{9}$ \\
\hline \multicolumn{5}{|l|}{$\begin{array}{l}\text { Surrogate NAb } \\
\text { Mean (AU/mL) }{ }^{1}\end{array}$} \\
\hline Baseline & $4.1(3.9-4.3)$ & $4.0(4.0-4.0)$ & $4.1(3.9-4.4)$ & 0.610 \\
\hline Day21 2/Day28 & $37.1(27.6-46.6)^{3}$ & $70.6(30.3-110.8)$ & $5.5(4.7-6.4)^{6}$ & 0.001 \\
\hline Day 56 & $40.5(24.6-56.4)$ & $594.7(509.9-679.5)$ & $38.8(27.9-49.6)^{7}$ & $<0.0001$ \\
\hline Month 6 & $8.6(7.2-9.9)^{4}$ & $138.5(80.5-196.6)^{5}$ & $9.2(3.5-14.9)^{8}$ & 0.001 \\
\hline \multicolumn{5}{|l|}{ Anti-N IgG } \\
\hline Baseline & $1.4(1.0-1.9)$ & $1.7(0.6-2.8)$ & $0.8(0.6-1.0)$ & 0.166 \\
\hline Day21 2 /Day28 & $1.4(0.9-1.8)^{3}$ & $1.6(0.6-2.6)$ & $1.6(1.1-2.0)^{6}$ & 0.904 \\
\hline Day 56 & $1.4(0.9-1.8)$ & $1.3(0.4-2.2)$ & $21.8(15.8-27.8)^{7}$ & $<0.0001$ \\
\hline Month 6 & $1.3(0.7-1.9)^{4}$ & $1.3(0.4-2.2)^{5}$ & $5.9(-4.8-16.7)^{8}$ & 0.007 \\
\hline & \multicolumn{4}{|c|}{$\begin{array}{l}\text { 1: data are mean values }(95 \% \mathrm{CI}){ }^{2}: \text { the blood samples of the BNT162 group collected on day } 21 .{ }^{3}: 41 \text { individual } \\
\text { collected blood samples on day } 28 .{ }^{4}: 28 \text { individuals collected blood samples at } 6 \text { months. }{ }^{5}: 38 \text { individual } \\
\text { collected blood samples at } 6 \text { months. }{ }^{6}: 40 \text { individuals collected blood samples on day } 28 .{ }^{7}: 38 \text { individual } \\
\text { collected blood samples on day } 56 .{ }^{8}: 4 \text { individuals collected blood samples at } 6 \text { months. }{ }^{9}: p<0.05 \text {, the results are } \\
\text { significantly different. }\end{array}$} \\
\hline
\end{tabular}

Table 4. Adverse events.

\begin{tabular}{|c|c|c|c|c|}
\hline & Combo $(n=42)$ & BNT162b2 $(n=33)$ & CoronaVac $(n=35)$ & $p^{2}$ \\
\hline $\begin{array}{l}\text { Post first dose } \\
\text { System reactions }\end{array}$ & $19(45.2 \%)$ & $15(45.5 \%)$ & $13(37.1 \%)$ & 0.761 \\
\hline Fever & $2(4.8 \%)$ & $1(3.0 \%)$ & $0(0)$ & 0.636 \\
\hline Chills & $0(0)$ & $0(0)$ & $2(5.7 \%)$ & 0.187 \\
\hline Headache & $6(14.3 \%)$ & $6(18.2 \%)$ & $4(11.4 \%)$ & 0.702 \\
\hline Tiredness & $12(31.0 \%)$ & $11(33.3 \%)$ & $9(25.7 \%)$ & 0.807 \\
\hline Nausea & $1(2.4 \%)$ & $1(3.0 \%)$ & $3(8.6 \%)$ & 0.516 \\
\hline Vomit & $0(0)$ & $0(0)$ & $0(0)$ & - \\
\hline Diarrhea & $1(2.4 \%)$ & $2(6.1 \%)$ & $2(5.7 \%)$ & 0.732 \\
\hline Muscle pain & $7(16.7 \%)$ & $9(27.3 \%)$ & $6(17.1 \%)$ & 0.500 \\
\hline Joint pain & $1(2.4 \%)$ & $4(12.1 \%)$ & $2(5.7 \% 0$ & 0.255 \\
\hline Skin rash & $3(7.1 \%)$ & $1(3.0 \%)$ & $1(2.9 \%)$ & 0.624 \\
\hline $\mathrm{SAE}^{1}$ & $0(0)$ & $0(0)$ & $0(0)$ & - \\
\hline Local symptoms & $41(97.6 \%)$ & $27(81.8 \%)$ & $12(34.3 \%)$ & $<0.0001$ \\
\hline Pain & $41(97.6 \%)$ & $25(75.8 \%)$ & $12(34.3 \%)$ & $<0.0001$ \\
\hline Redness & $3(7.1 \%)$ & $7(21.2 \%)$ & $0(0)$ & 0.005 \\
\hline Swelling & $6(14.3 \%)$ & $12(36.4 \%)$ & $0(0)$ & $<0.0001$ \\
\hline Ecchymosis & $4(9.5 \%)$ & $3(9.1 \%)$ & $0(0)$ & 0.200 \\
\hline Itching & $2(4.8 \%)$ & $3(9.1 \%)$ & $1(2.9 \%)$ & 0.200 \\
\hline
\end{tabular}


Table 4. Cont.

\begin{tabular}{ccccc}
\hline & Combo $(\boldsymbol{n}=\mathbf{4 2})$ & BNT162b2 $(\boldsymbol{n}=\mathbf{3 3})$ & CoronaVac $(\boldsymbol{n}=\mathbf{3 5})$ & $p^{\mathbf{2}}$ \\
\hline Post second dose & & & & $<0.0001$ \\
System reactions & $13(31.0 \%)$ & $22(66.7 \%)$ & $8(22.9 \%)$ & 0.088 \\
Fever & $0(0)$ & $2(6.1 \%)$ & $0(0)$ & 0.002 \\
Chills & $0(0)$ & $6(18.2 \%)$ & $1(2.9 \%)$ & 0.107 \\
Headache & $6(14.3 \%)$ & $9(27.3 \%)$ & $3(8.6 \%)$ & $<.0001$ \\
Tiredness & $10(23.8 \%)$ & $17(51.5 \%)$ & $2(8.6 \%)$ & 0.233 \\
Nausea & $2(4.8 \%)$ & $5(15.2 \%)$ & $1(5.7 \%)$ & 0.524 \\
Vomit & $0(0)$ & $1(3.0 \%)$ & $3(8.6 \%)$ & 0.157 \\
Diarrhea & $0(0)$ & $3(9.1 \%)$ & $3(8.6 \%)$ & 0.0001 \\
Muscle pain & $3(7.1 \%)$ & $16(48.5 \%)$ & $1(2.9 \%)$ & 0.046 \\
Joint pain & $1(2.4 \%)$ & $5(15.2 \%)$ & $0(0)$ & 0.300 \\
Skin rash & $0(0)$ & $1(3.0 \%)$ & $0(0)$ & - \\
SAE $\mathbf{1}$ & $0(0)$ & $0(0)$ & $7(20.0 \%)$ & $<0.0001$ \\
Local symptoms & $15(35.7 \%)$ & $25(75.8 \%)$ & $7(20.0 \%)$ & $<0.0001$ \\
Pain & $15(35.7 \%)$ & $23(69.7 \%)$ & $1(2.9 \%)$ & 0.001 \\
Redness & $2(4.8 \%)$ & $9(27.3 \%)$ & $0(0)$ & $<.0001$ \\
Swelling & $2(4.8 \%)$ & $11(33.3 \%)$ & $0(0)$ & 0.300 \\
Ecchymosis & $0(0)$ & $1(3.0 \%)$ & $0(0)$ & 0.002 \\
Itching & $0(0)$ & $5(15.2 \%)$ & & \\
\hline
\end{tabular}

1: SAE: severe adverse events, vaccine-related undesired events including death, disability or life-threatening conditions. ${ }^{2}: p<0.05$, the results are significantly different.

\section{Discussion}

In this study, the efficacy of three vaccination strategies against SARS-CoV-2 was determined. In the vMN assay with WT, the Combo group showed higher immunogenicity than other groups after the first dose, while the BNT162b2 platform showed the highest immunogenicity after full vaccination. When the serum samples were tested again in different variants, we found that the antibody titers reduced, especially against the beta and theta variants. After 6 months of vaccination, the neutralizing activity dropped further in the WT and all variants.

Comparing the immunogenicity of the three strategies against WT, BNT162b2 showed significantly higher efficacy after full vaccination (day 56 GMT, 136.9), and the serum samples of subjects vaccinated with the Combo vaccine (day 56 GMT, 26.2) also showed higher efficacy than CoronaVac (day 56 GMT, 14.7) (Table 1). The development of BNT162b2 and CoronaVac is based on two different platforms. The CoronaVac vaccine involves inactivated viruses to activate humoral immunity, while the BNT162b2 vaccine works via translating targeted antigens to induce an immune response in the host [19-21]. As the immunogenicity of vaccines from various platforms is different after vaccination, heterogeneous prime-boost vaccination that combines different platforms may produce comparable efficacy with, or even higher efficacy than, the homologous CoronaVac vaccine [2,3]. In one clinical study, Vasileiou et al. reported that the effectiveness of primer-dose BNT162b2 was $85 \%$ at day $28-34$ post-vaccination and then dropped to a level of $64 \%$ after 42 days post-vaccination [22]. Furthermore, from our previous study, the neutralizing activity in recovered patients with previous COVID-19 infection decreased significantly at week 6 after discharge [23]. Thus, the antibody response on day 56 might be lower than on day 28 in healthy individuals who have just received one dose of BNT162b2. In this study, the day 56 GMT was comparable to the day 28 GMT in the Combo group. Despite an inferior neutralizing antibody response when compared to the homologous BNT162b2, one dose of the CoronaVac vaccine could maintain the immune response induced by the primer dose of BNT162b2 in the Combo group. Studies on the mRNA vaccines have demonstrated their high clinical effectiveness and are adopted widely throughout the world [24,25]. However, the shortage of mRNA COVID-19 vaccines in some countries resulted in the delay of the second dose [26]. For these individuals who cannot receive the second dose of the BNT162b2 vaccine on time, they can opt for one dose of CoronaVac to complete the vaccination regime. 
Furthermore, subjects who cannot tolerate the side effects induced by the second dose of the BNT162b2 vaccine can also switch to one dose of CoronaVac after priming with the BNT162b2 (Table 4).

The various mutation of the SARS-CoV-2 has caused concerns regarding the effectiveness of the current vaccines; therefore, we have tested antibodies against the main variants. The results showed that the vMN titre all decreased for the D614G, beta, theta and delta variants. Compared with Combo and CoronaVac vaccine, BNT162b2 still showed better immunogenicity against the variant virus after two doses. The Combo vaccine has not elicited better neutralizing activity against variants than the CoronaVac. Interestingly, a reduction of GMT on day 56 and month 6 against alpha was observed in the Combo and CoronaVac groups when compared with that against WT, but the neutralizing activity against alpha and WT was similar after two doses of BNT162b2 (Table 2). The reason may be that mutation in the alpha variant caused the escape of the virus from the antibody response induced by the CoronaVac vaccine $[27,28]$. It has been reported that $31.8 \%$ and $59.1 \%$ of plasma from individuals receiving the CoronaVac vaccine failed to detect the effective neutralization of alpha and gamma variants. Furthermore, our study found that the performance of these vaccines in beta and theta variants was inferior to other variants. Both the beta and theta variants contain N501Y and E484K mutations in the S-protein, which enhanced the affinity of ACE2, and immune sera showed decreased activity when the mutations, including N501Y and E484K [6,10,29]. Although the delta variant is more contagious, BNT162b2 still showed satisfactory efficacy after two doses (day 56 GMT, 74.6). A study has shown that the effectiveness of two doses of BNT162b2 was $88.0 \%$ among the vaccinated population with the delta variant, while $93.7 \%$ among those with alpha variant, but the effectiveness against the delta variant was significantly lower than that of the alpha variant after one dose [30].

Interestingly, the vMN test for WT performed on the participants before the second dose showed that the vMN titer of the Combo group was higher than that of the BNT162b2 group (23.7 vs. 13.4), even though the primer dose of the two groups were the same. The difference lies in the interval between vaccination. The Combo group assay was taken on day 28, while the BNT162b2 group was on day 21. It may indicate that after the first injection of the mRNA vaccine, the immunogenicity has not reached the peak value in 21 days; thereby the delay of the second dose of the BNT162b2 vaccine may have a better antibody response. Currently, there is no comparative study on the vaccine interval of BNT162b2, but in the ChAdOx1 nCoV-19 vaccine study, it has shown higher efficacy with a vaccination interval over six weeks [4]. Meanwhile, delaying the second dose might offer a good strategy to tackle vaccine shortages and could expand the vaccine coverage, especially in the high-risk population [31].

Moreover, the neutralizing protection induced by vaccines in three groups dropped a lot at 6 months when compared to that on day 56. For delta, month 6 GMT decreased from 7.4 to 5.5 in the Combo group, from 74.6 to 22.7 in the BNT162b2 group, and from 8.0 to 5 in the CoronaVac group (Table 2). For WT, the month 6 GMT was only 8.0, 28.8 and 7.1 in the Combo, BNT162b2, and CoronaVac groups, respectively. Some studies also reported a reduction in the effectiveness of COVID-19 at 6 months $[7,8]$. One clinical study conducted in the USA reported that the BNT162b2 vaccine effectiveness against delta decreased to 53\% after 4 months when compared to $93 \%$ during the first month after two doses of vaccine [7]. Thus, the booster dose should be administered to these individuals receiving BNT162b2 at 5 months post-second dose, while people vaccinated with the BNT162b2-CoronaVac combination or CoronaVac should receive the third dose earlier. The BNT162b2 vaccine would be the preferred COVID-19 booster, according to these study results.

The limitation of the study is the lack of sera collected between day 56 and month 6 , and as a result, could not determine the optimal time to receive the third dose of vaccine. The time of the sera collection after the first dose was different between the BNT162b2 group and the Combo and CoronaVac groups due to the time restriction for the second dose for the respective vaccine platforms. We were unable to test the various vaccine 
platforms, especially the Combo platform, in preventing COVID-19 infection, as there were very few imported COVID-19 patients and no local patients over the previous 6 months due to the very strict quarantine policy imposed on the returned travelers. Besides, the immunogenicity of CoronaVac as a primer followed by one dose of the BNT162b2 vaccine deserves further investigation, and we have began to recruit volunteers to receive the heterologous CoronaVac-BNT162b2 vaccination strategy.

\section{Conclusions}

In this study, heterologous vaccination strategy was safe. The overall vaccine immunogenicity of the Combo platform at day 56 and 6 months was inferior to the homologous BNT162b2 platform but comparable to the homologous CoronaVac platform, against both WT and all variants. Furthermore, the neutralizing activity against SARS-CoV-2 waned at 6 months regardless of the three vaccination platforms. Thus, it is recommended that one booster of COVID-19 vaccine should be administered at 5 months after completing the second dose. Furthermore, the mix-and-match approach for the booster shot requires further investigation regarding people who have already received two doses of the COVID-19 vaccines.

Author Contributions: Conceptualization, I.F.-N.H., K.-H.C., H.C. and K.-Y.Y.; methodology, R.Z., K.-Y.L., D.L., Y.F. and L.L.; validation, R.Z., K.-Y.L. and D.L.; formal analysis, R.Z., K.-Y.L. and D.L.; investigation, R.Z., K.-Y.L., D.L., Y.F., L.L. and P.-C.C.; data curation, R.Z. and D.L.; writing-original draft preparation, R.Z. and D.L.; writing-review and editing, I.F.-N.H. and K.-H.C.; project administration, I.F.-N.H. and K.-H.C.; supervision, I.F.-N.H., K.-H.C., K.K.-W.T., H.C. and K.-Y.Y. All authors have read and agreed to the published version of the manuscript.

Funding: The study was supported by the Health and Medical Research Fund, Commissioned Research on the Novel Coronavirus Disease (COVID-19)(Grant number: COVID190124 and COVID190125; Funder: Food and Health Bureau, HKSAR).

Institutional Review Board Statement: The study was conducted according to the guidelines of the Declaration of Helsinki and approved by the Institutional Review Board of the University of Hong Kong/Hospital Authority Hong Kong West Cluster (UW 21-214, 22 March 2021).

Informed Consent Statement: Informed consent was obtained from all subjects involved in the study.

Data Availability Statement: The data used to support the findings of this study are included within the article.

Acknowledgments: We sincerely thank all staff and students who were involved in the study.

Conflicts of Interest: The authors declare no conflict of interest.

\section{References}

1. World Health Organization. Coronavirus Disease 2019 (COVID-19) Situation Report. Available online: https://www.who.int/ emergencies / diseases / novel-coronavirus-2019 (accessed on 23 November 2021).

2. Polack, F.P.; Thomas, S.J.; Kitchin, N.; Absalon, J.; Gurtman, A.; Lockhart, S.; Perez, J.L.; Pérez Marc, G.; Moreira, E.D.; Zerbini, C.; et al. Safety and Efficacy of the BNT162b2 mRNA COVID-19 Vaccine. N. Engl. J. Med. 2020, 383, $2603-2615$. [CrossRef] [PubMed]

3. Tanriover, M.D.; Doğanay, H.L.; Akova, M.; Güner, H.R.; Azap, A.; Akhan, S.; Köse, Ş.; Erdinç, F.; Akalın, E.H.; Tabak, Ö.F.; et al. Efficacy and safety of an inactivated whole-virion SARS-CoV-2 vaccine (CoronaVac): Interim results of a double-blind, randomised, placebo-controlled, phase 3 trial in Turkey. Lancet 2021, 398, 213-222. [CrossRef]

4. Voysey, M.; Clemens, S.A.C.; Madhi, S.A.; Weckx, L.Y.; Folegatti, P.M.; Aley, P.K.; Angus, B.; Baillie, V.L.; Barnabas, S.L.; Bhorat, Q.E.; et al. Safety and efficacy of the ChAdOx1 nCoV-19 vaccine (AZD1222) against SARS-CoV-2: An interim analysis of four randomised controlled trials in Brazil, South Africa, and the UK. Lancet 2021, 397, 99-111. [CrossRef]

5. Heath, P.T.; Galiza, E.P.; Baxter, D.N.; Boffito, M.; Browne, D.; Burns, F.; Chadwick, D.R.; Clark, R.; Cosgrove, C.; Galloway, J.; et al. Safety and Efficacy of NVX-CoV2373 COVID-19 Vaccine. N. Engl. J. Med. 2021, 385, 1172-1183. [CrossRef]

6. Tregoning, J.S.; Flight, K.E.; Higham, S.L.; Wang, Z.; Pierce, B.F. Progress of the COVID-19 vaccine effort: Viruses, vaccines and variants versus efficacy, effectiveness and escape. Nat. Rev. Immunol. 2021, 21, 626-636. [CrossRef] 
7. Tartof, S.Y.; Slezak, J.M.; Fischer, H.; Hong, V.; Ackerson, B.K.; Ranasinghe, O.N.; Frankland, T.B.; Ogun, O.A.; Zamparo, J.M.; Gray, S.; et al. Effectiveness of mRNA BNT162b2 COVID-19 vaccine up to 6 months in a large integrated health system in the USA: A retrospective cohort study. Lancet 2021, 398, 1407-1416. [CrossRef]

8. Thomas, S.J.; Moreira, E.D., Jr.; Kitchin, N.; Absalon, J.; Gurtman, A.; Lockhart, S.; Perez, J.L.; Perez Marc, G.; Polack, F.P.; Zerbini, C.; et al. Safety and Efficacy of the BNT162b2 mRNA COVID-19 Vaccine through 6 Months. N. Engl. J. Med. 2021, 385, 1761-1773. [CrossRef] [PubMed]

9. Hou, Y.J.; Chiba, S.; Halfmann, P.; Ehre, C.; Kuroda, M.; Dinnon, K.H., 3rd; Leist, S.R.; Schäfer, A.; Nakajima, N.; Takahashi, K.; et al. SARS-CoV-2 D614G variant exhibits efficient replication ex vivo and transmission in vivo. Science 2020, 370, 1464-1468. [CrossRef]

10. Gómez, C.E.; Perdiguero, B.; Esteban, M. Emerging SARS-CoV-2 Variants and Impact in Global Vaccination Programs against SARS-CoV-2/COVID-19. Vaccines 2021, 9, 243. [CrossRef] [PubMed]

11. Mohammadi, M.; Shayestehpour, M.; Mirzaei, H. The impact of spike mutated variants of SARS-CoV2 [Alpha, Beta, Gamma, Delta, and Lambda] on the efficacy of subunit recombinant vaccines. Braz. J. Infect. Dis. 2021, 25, 101606. [CrossRef]

12. Shinde, V.; Bhikha, S.; Hoosain, Z.; Archary, M.; Bhorat, Q.; Fairlie, L.; Lalloo, U.; Masilela, M.S.L.; Moodley, D.; Hanley, S.; et al. Efficacy of NVX-CoV2373 COVID-19 Vaccine against the B.1.351 Variant. N. Engl. J. Med. 2021, 384, 1899-1909. [CrossRef]

13. Madhi, S.A.; Baillie, V.; Cutland, C.L.; Voysey, M.; Koen, A.L.; Fairlie, L.; Padayachee, S.D.; Dheda, K.; Barnabas, S.L.; Bhorat, Q.E.; et al. Efficacy of the ChAdOx1 nCoV-19 COVID-19 Vaccine against the B.1.351 Variant. N. Engl. J. Med. 2021, 384, 1885-1898. [CrossRef] [PubMed]

14. Lustig, Y.; Zuckerman, N.; Nemet, I.; Atari, N.; Kliker, L.; Regev-Yochay, G.; Sapir, E.; Mor, O.; Alroy-Preis, S.; Mendelson, E.; et al. Neutralising capacity against Delta (B.1.617.2) and other variants of concern following Comirnaty (BNT162b2, BioNTech/Pfizer) vaccination in health care workers, Israel. Eurosurveill 2021, 26, 2100557. [CrossRef]

15. Pollard, A.J.; Launay, O.; Lelievre, J.D.; Lacabaratz, C.; Grande, S.; Goldstein, N.; Robinson, C.; Gaddah, A.; Bockstal, V.; Wiedemann, A.; et al. Safety and immunogenicity of a two-dose heterologous Ad26.ZEBOV and MVA-BN-Filo Ebola vaccine regimen in adults in Europe (EBOVAC2): A randomised, observer-blind, participant-blind, placebo-controlled, phase 2 trial. Lancet Infect. Dis. 2021, 21, 493-506. [CrossRef]

16. Ledford, H. Could mixing COVID vaccines boost immune response? Nature 2021, 590, 375-376. [CrossRef]

17. Hillus, D.; Schwarz, T.; Tober-Lau, P.; Vanshylla, K.; Hastor, H.; Thibeault, C.; Jentzsch, S.; Helbig, E.T.; Lippert, L.J.; Tscheak, P.; et al. Safety, reactogenicity, and immunogenicity of homologous and heterologous prime-boost immunisation with ChAdOx1 nCoV-19 and BNT162b2: A prospective cohort study. Lancet Respir. Med. 2021. [CrossRef]

18. Chan, K.H.; Leung, K.Y.; Zhang, R.R.; Liu, D.; Fan, Y.; Chen, H.; Yuen, K.Y.; Hung, I.F. Performance of a Surrogate SARS-CoV-2Neutralizing Antibody Assay in Natural Infection and Vaccination Samples. Diagnostics 2021, 11, 1757. [CrossRef]

19. Park, J.W.; Lagniton, P.N.P.; Liu, Y.; Xu, R.H. mRNA vaccines for COVID-19: What, why and how. Int. J. Biol. Sci. 2021, 17, 1446-1460. [CrossRef]

20. Zhang, Y.; Zeng, G.; Pan, H.; Li, C.; Hu, Y.; Chu, K.; Han, W.; Chen, Z.; Tang, R.; Yin, W.; et al. Safety, tolerability, and immunogenicity of an inactivated SARS-CoV-2 vaccine in healthy adults aged 18-59 years: A randomised, double-blind, placebocontrolled, phase $1 / 2$ clinical trial. Lancet Infect. Dis. 2021, 21, 181-192. [CrossRef]

21. Walsh, E.E.; Frenck, R.W., Jr.; Falsey, A.R.; Kitchin, N.; Absalon, J.; Gurtman, A.; Lockhart, S.; Neuzil, K.; Mulligan, M.J.; Bailey, R.; et al. Safety and Immunogenicity of Two RNA-Based COVID-19 Vaccine Candidates. N. Engl. J. Med. 2020, 383, 2439-2450. [CrossRef]

22. Vasileiou, E.; Simpson, C.R.; Shi, T.; Kerr, S.; Agrawal, U.; Akbari, A.; Bedston, S.; Beggs, J.; Bradley, D.; Chuter, A.; et al. Interim findings from first-dose mass COVID-19 vaccination roll-out and COVID-19 hospital admissions in Scotland: A national prospective cohort study. Lancet 2021, 397, 1646-1657. [CrossRef]

23. Zhang, R.; Khong, K.W.; Leung, K.Y.; Liu, D.; Fan, Y.; Lu, L.; Chan, P.C.; Chen, L.; Kelvin Kai-Wang To, K.K.W.; Chen, H.; et al. Antibody Response of BNT162b2 and CoronaVac Platforms in Recovered Individuals Previously Infected by COVID-19 against SARS-CoV-2 Wild Type and Delta Variant. Vaccines 2021, 9, 1442. [CrossRef]

24. Kon, E.; Elia, U.; Peer, D. Principles for designing an optimal mRNA lipid nanoparticle vaccine. Curr. Opin. Biotechnol. 2021, 73, 329-336. [CrossRef]

25. Thomas, S.J.; Moreira, E.D., Jr.; Kitchin, N.; Absalon, J.; Gurtman, A.; Lockhart, S.; Perez, J.L.; Marc, G.P.; Polack, F.P.; Zerbini, C.; et al. Six Month Safety and Efficacy of the BNT162b2 mRNA COVID-19 Vaccine. MedRxiv 2021, 21. [CrossRef]

26. Miron, O.; Wilf-Miron, R.; Davidovitch, N. Effectiveness of COVID-19 vaccines BNT162b2 and mRNA-1273 by days from vaccination: A reanalysis of clinical trial data. SSRN 2021, 14. [CrossRef]

27. Fernández, J.; Bruneau, N.; Fasce, R.; Martín, H.S.; Balanda, M.; Bustos, P.; Ulloa, S.; Mora, J.; Ramírez, E. Neutralization of alpha, gamma, and D614G SARS-CoV-2 variants by CoronaVac vaccine-induced antibodies. J. Med. Virol. 2021, 94, 399-403. [CrossRef]

28. Xie, X.; Liu, Y.; Liu, J.; Zhang, X.; Zou, J.; Fontes-Garfias, C.R.; Xia, H.; Swanson, K.A.; Cutler, M.; Cooper, D.; et al. Neutralization of SARS-CoV-2 spike 69/70 deletion, E484K and N501Y variants by BNT162b2 vaccine-elicited sera. Nat. Med. 2021, 27,620-621. [CrossRef] [PubMed] 
29. Wibmer, C.K.; Ayres, F.; Hermanus, T.; Madzivhandila, M.; Kgagudi, P.; Oosthuysen, B.; Lambson, B.E.; de Oliveira, T.; Vermeulen, M.; van der Berg, K.; et al. SARS-CoV-2 501Y.V2 escapes neutralization by South African COVID-19 donor plasma. Nat. Med. 2021, 27, 622-625. [CrossRef]

30. Lopez Bernal, J.; Andrews, N.; Gower, C.; Gallagher, E.; Simmons, R.; Thelwall, S.; Stowe, J.; Tessier, E.; Groves, N.; Dabrera, G.; et al. Effectiveness of COVID-19 Vaccines against the B.1.617.2 (Delta) Variant. N. Engl. J. Med. 2021, 385, 585-594. [CrossRef] [PubMed]

31. Iacobucci, G.; Mahase, E. COVID-19 vaccination: What's the evidence for extending the dosing interval? Bmj $2021,372, \mathrm{n} 18$. [CrossRef] 